\title{
Aplicación del GIS en la caracterización edafoclimática, del distrito de Cacatachi - provincia de San Martín, con fines de producción agrícola rentable y sostenible
Application of GIS in the edaphoclimatic characterization of the Cacatachi district - San Martín province, for profitable and sustainable agricultural production purposes \\ Castillo-Diaz, Tedy ${ }^{1 \text { [0000-0002-4802-9115] }}$; Lopez-Fulca, Roaldo ${ }^{1[0000-0002-2333-}$ ${ }^{253 X]}$ y Ramirez-Navarro, Williams 1 [0000-0002-8165-094X] \\ ${ }^{1}$ Universidad Nacional de San Martín, Tarapoto, Perú tcastillodunsm.edu.pe
}

Resumen. La caracterización edafoclimática de los suelos es un proceso que contribuye a la planificación de las siembras con mejores perspectivas rentables, siendo el punto de partida de la actividad productiva agropecuaria. El objetivo fue generar una base de datos geoespaciales integrada de condiciones edafoclimáticas del distrito de Cacatachi, de consulta dinámica a través de mapas temáticos como de fisiografía, capacidad de uso, características físicas y químicas de suelos y de uso actual de las tierras. Para ello se empleó un software GIS utilizando información de instituciones públicas que iniciaron el proceso; asimismo, la metodología de trabajo tuvo dos etapas, a nivel de gabinete y a nivel de campo. El resultado fue la base de datos Quantum GIS (QGIS), que facilita el manejo de la información edafoclimática, con 104 mapas en detalle, cuyas representaciones espaciales exponen la caracterización de las zonas estratificadas en el proyecto, donde se hicieron las calicatas, de los cuales se hicieron los análisis químicos, físicos y biológicos de los horizontes identificados en los perfiles. La información edafoclimática generada nos aproxima a una microzonificación en detalle, que se convierte en herramienta orientadora de las siembras de cultivos, en función a las potencialidades nutricionales, fisiográficas y climáticas de los suelos, segmentados en sectores productivos.

Palabras clave: Caracterización edafoclimática, mapas temáticos, productividad, programa Quantum GIS

Citar como: Castillo-Diaz, T., Lopez-Fulca, R., \& Ramirez-Navarro, W. (2021). Aplicación del GIS en la caracterización edafoclimática, del distrito de Cacatachi - provincia de San Martín, con fines de producción agrícola rentable y sostenible. Revista Agrotecnológica Amazónica, 1(2), 53-67. https://doi.org/10.51252/ra a.v1i2.192

Recibido: 20/04/2020

Aceptado: 20/06/2020

Publicado: 19/07/2021
Abstract. The edaphoclimatic characterization of soils is a process that contributes to the planning of plantings with better profitable prospects, being the starting point of agricultural production activity. The objective was to generate an integrated geospatial database of edaphoclimatic conditions of the Cacatachi district, for dynamic consultation through thematic maps such as physiography, use capacity, physical and chemical characteristics of soils and current land use. For this, GIS software was used using information from public institutions that started the process; Likewise, the work methodology had two stages, at the cabinet level and at the field level. The result was the Quantum GIS database (QGIS), which facilitates the management of edaphoclimatic information, with 104 detailed maps, whose spatial representations expose the characterization of the stratified areas in the project, where the pits were made, of the which were made the chemical, physical and biological analyses of the horizons identified in the profiles. The edaphoclimatic information generated brings us closer to a micro-zoning in detail, which becomes a guiding tool for planting crops, based on the nutritional, physiographic and climatic potential of the soils, segmented into productive sectors.

Keywords: Edaphoclimatic characterization, thematic maps, productivity, Quantum GIS software 


\section{$1 \quad$ Introducción}

La agricultura es una de las principales actividades económicas por los altos productivos (Stojanovic, 2019), además representa un papel muy importante en la sostenibilidad de un país (Rizaldi et al., 2019), por otra parte, es el encargado de preservar la seguridad alimentaria a nivel internacional (Lesmo Duarte et al., 2018). Sin embargo, la seguridad alimentaria se está convirtiendo en una problemática de gran importancia, debido a factores como la industrialización y la rápida urbanización, que están agotando los recursos hídricos y las tierras (Lyford, 2017).

La agricultura se encuentra en el sector más crítico en cuanto a la producción de alimentos, frente a la inminente amenaza, depender de métodos tradicionales es inminente para que el hombre pueda satisfacer sus necesidades básicas (Nabati et al., 2020). Es por ello, que la adopción de nuevos mecanismos o herramientas tecnológicas son fundamentales para maximizar los recursos naturales que van en disminución (Mok et al., 2020), y sobre todo que se contribuya al desarrollo de la agricultura sostenible, teniendo como bases antecedentes y barreras existentes en dicho sector (Laurett et al., 2021).

Smart Farming (agricultura inteligente) es un término nuevo en el sector agrícola, cuyo objetivo es reemplazar las técnicas tradicionales en soluciones basadas en Tecnologías de la Información y Comunicación (TIC), para aumentar la producción, reducir costos en insumos y mejorar la calidad de los productos (Moysiadis et al., 2021). Los Sistemas de Información Geográfica (GIS), es una de las herramientas que proporciona recursos de información geográficos que sirven como apoyo al sector agrícola para el trabajo en las tierras (Longley \& Frank Goodchild, 2020). Además, Kahveci (2017) afirma que, el uso de las TIC, la teledetección, GIS y GNSS en la agricultura va en aumento gradual en los países desarrollados.

En el entorno agrícola existe la variabilidad espacial conjunta del suelo y el clima, ofreciendo así la oportunidad de delimitar zonas edafoclimáticas, que servirán para mejorar la gestión de los recursos. Asimismo, Nabati et al. (2020) sostienen que, la zonificación de tierras con respecto al clima, topografía, suelo y relieve son características que influyen en la producción de un determinado producto, es por ello que, Perez-Garcia et al. (2019) afirman que los GIS posibilitan la clasificación y mapeo de cultivos con información georreferenciada, además, ayudan a optimizar la producción utilizando mejores prácticas de gestión (Mani et al., 2021).

En Indonesia, se creó un mapa de equilibrio de uso de la tierra/cobertura de la tierra (LULC) basado en SIG, utilizaron imágenes de satélite 7 ETM y mapas activos con el fin de evaluar los cambios en el uso de las tierras. Los resultados del procesamiento de las imágenes de campo fueron basados en los criterios del coeficiente de Cohen Kappa, obteniendo precisión e 
idoneidad. Se concluye que los datos geoespaciales obtenido en el mapa de equilibrio de LULC son esenciales para la toma de decisiones que planifiquen el desarrollo regional de la provincia de Sulawesi, Indonesia (Saing et al., 2021).

En la región Lambayeque, Perú el Instituto Nacional de Innovación Agraria (INIA) desarrollo un SIG denominado PETEFA, está herramienta proporciona información sobre las estaciones meteorológicas, tipo de suelo, usos de la tierra y georreferenciación, asimismo ofrece información temporal sobre la salud de los cultivos a lo largo de su vida, además la versión móvil permite que los agricultores de mantengan informados sobre el estado de cada una de sus parcelas (Palomino et al., 2018).

En el entorno local la región San Martín expone actualmente un vertiginoso desarrollo de la producción agropecuaria, destacando el despegue con cultivos como el café, cacao, palma aceitera, cítricos, sacha inchi, etc, cuya expansión de sus áreas, lo ubica a nivel del Perú como una región abastecedora de alimentos, y como tal, hay importancia de lineamientos sobre estos cultivos, sobre todo en los temas de mejoramiento genético, extensión en labores agronómicas, en post cosecha, y sobre todo en la obtención de rendimiento rentables de los cultivos (GORESAM, 2020).

En los últimos años ha cobrado importancia el concepto de la caracterización edafoclimática, de los territorios, entendiéndose las micro zonificaciones a nivel del suelo y de las condiciones del clima, en las zonas o distritos altamente productivos (Aguirre-Forero et al., 2018). En San Martín, el Instituto de Investigación de la Amazonía Peruana (IIAP), el Gobierno Regional y algunas municipalidades distritales, ya iniciaron este fenomenal proceso, desde el año 2004, en niveles de macro y meso zonificación, mas no en niveles micro, que son herramientas de planificación, que hace falta a los gobiernos locales con amplios potenciales de desarrollos rurales (GORESAM, 2009).

La caracterización edafoclimática de los suelos, expone una gran importancia, desde el punto de vista de la planificación de las siembras y cosechas, con criterios de orientación hacia cosechas rentables y para tomar decisiones a partir de la referenciación geográfica de la calidad agroecológica que se tiene de los suelos y de sus condiciones climáticas que presentan las zonas productoras (Giannini Kurina et al., 2018), como proyecto piloto, se estudió el área geográfica del distrito de Cacatachi, en función a un programa GIS, que muestra los contenidos edafoclimáticas, en base a los pisos altitudinales y capacidad de uso de los suelos, con los señalamientos técnicos y con sus respectivos potenciales productivos.

Este estudio tiene dos fases con los siguientes objetivos. Fase 1: utilizar un programa libre de SIG el para el análisis espacial del distrito de Cacatachi - provincia de San Martín, para generar de una base de datos geoespaciales (acondicionamiento cartográfico), que permita consultar de 
manera fácil a través de una tabla de atributos las diferentes características físicas de la zona de interés; el acondicionamiento cartográfico temático de suelos, fisiografía, capacidad de uso mayor de los suelos y uso actual de la tierra. Fase 2: diseñar e implementar un aplicativo geográfico a nivel de escritorio, que almacene información temática de las características edafoclimáticas del distrito de Cacatachi.

La investigación se justifica debido a la importancia del tema en estudio; bajo este contexto Zhang \& Cao (2019) infieren que, la aplicación de los GIS en la agricultura es un campo de investigación nuevo e importante, pues ayuda al desarrollo sostenible, planificación y gestión de los campos agrícolas, asimismo Sun et al. (2018) mencionan que los mapas temáticos de información agrícola reflejan el estado actual de la agricultura en una región y genera valor con referencia a la formulación de políticas agrícola y planes económicos.

\section{Materiales y Métodos}

\subsection{Nivel de gabinete}

\section{Fase I}

Diseño de la investigación: se determinaron los objetivos, productos, disponibilidad de tiempo y recursos financieros. Seguidamente se realizó la evaluación de la información, y viabilidad de la investigación. Posterior se definió el plan general, cronograma de actividades, presupuesto, la orientación de la base de datos y el nivel de estudio.

El inventario y recopilación de la información espacial y numérica consistió en recabar informes, reportes, mapas e imágenes, para propósitos de la zonificación de los cultivos, de las diferentes instituciones y por elaboración propia obteniendo: mapa topográfico, mapa de suelos y pendiente, mapa de temperatura mínima media absoluta anual, mapa de temperatura máxima media absoluta, mapa de isoyetas (precipitación total anual).

Técnica de recopilación de Información climática: se recopiló información climatológica de temperaturas medias, máximas y mínimas y los valores pluviométricos de los años hidrológicos húmedos medios y secos de la estación meteorológica $\mathrm{CO}$ - Tarapoto conducido por el SENAMHI San Martín, permitiendo utilizarlos para la caracterización edafoclimática del distrito de Cacatachi.

Recopilación de material cartográfico: se recopiló información cartográfica base y temática a diferentes escalas como son: mapa edafológico, ecológico, fisiográfico, etc. para su posterior análisis y comparación con los requerimientos edafoclimáticos de los cultivos.

Generación de mapas temáticos: se adecuo los cortes cartográficos a la propuesta de límites referenciales (oficiales) del distrito de Cacatachi, así como la base de datos que le corresponde. 
Para este procedimiento cartográfico de composición de mapas o procesamiento de la base de datos para el distrito de Cacatachi, se realizó utilizando el programa QUANTUM GIS 2.4.; utilizando para ello los elementos de núcleo y otros complementos.

Cabe mencionar que en el presente trabajo no se generaron los mapas temáticos, éstos fueron elaborados en el proceso de meso ZEE de la Sub Cuenca del río Cumbaza.

\section{Fase II}

Metodología: cartográficamente, el presente trabajo cuenta con archivos tipo *. SHP, que representan los temáticos de suelo fisiográfica, uso actual capacidad de uso mayor de suelos referidas al distrito de Cacatachi, se tomó así mismos datos de la georreferenciación de los puntos de muestro de calicatas, estos datos son los principales insumos para realizar el aplicativo SIG, que muestra los temáticos y el análisis del suelo de las zonas muestreadas de Cacatachi.

Con los insumos vectorizados, se realizó el procesamiento y edición, con el uso del programa QGIS 2.4 y se definió el análisis espacial como parte del modelo conceptual, permitiendo definir las entidades del proyecto además de sus relaciones.

Posteriormente, se procedió a generar el modelo lógico el cual permitió establecer las tablas y sus atributos además crear las llaves primarias y las llaves de paso; que por la característica de la relación de las tablas que es de "muchos a muchos", y se adicionó una tabla de paso.

Para plasmar todo el proceso que se describió, en la creación de las tablas de la base de datos se empleó el programa PostgreSQL, sirviendo de plataforma para enlazar la información geográfica.

Especificación de la base de datos: entre los componentes primarios que se consideró en el diseño conceptual y físico de la base de datos tuvimos: capas temáticas, polígonos, líneas y puntos, se tiene:

Tablas de atributos y tablas de enlace a otras bases de datos, que consiste en el diseño tabular de los datos que se organiza con manejadores de base de datos relacionales, creando archivos espacio y mantenimiento, modificación y protección.

Anotaciones y diseño de la presentación de los resultados tanto gráfico y numérica. Se adaptó usar como herramienta de aplicación el Sistema de Información Geográfica (SIG) de código libre para plataformas GNU/Linux, Unix, Mac OS y Microsoft Windows. Permite manejar formatos raster y vectoriales a través de las bibliotecas GDAL y OGR, así como bases de datos. 


\subsection{Nivel de campo}

En base al mapa de capas fisiográficas y de Capacidad de Uso de los suelos, que se recopiló del distrito de Cacatachi, de fuentes de Instituto de Investigaciones de la Amazonía Peruana, de la ONERN y del Gobierno Regional de San Martín, se estratificó los sectores a ser estudiados, sus niveles de composición nutricional y de sus características químicas de los suelos, para tal efecto se geo referenció cada una de las zonas seccionadas entre sí, de tal manera que todo el área superficial del distrito fue analizado sus características de suelo.

El distrito de Cacatachi, según sus características fisiográficas y de capacidad de uso, se secciono en 6 sectores, en cada una de ellas se hizo las calicatas, para toma de datos.

Las calicatas tuvieron una dimensión de excavación de 1,2 m x 1,5 m x 1,50 m de profundidad.

Previo a la excavación de las calicatas, se hizo una descripción fisiográfica de los sectores estudiados, capturando su altitud respecto al nivel del mar, su composición vegetal y productiva.

En cada calicata se diferenció en horizontes, en cada horizonte visto en perfil, se extrajeron muestras representativas del suelo, que fueron llevadas al Laboratorio de Suelos del Facultad de Ciencias Agrarias de la UNSM donde se analizaron su textura, su pH, sus contenidos de N-P-K.

Con los resultados obtenidos en el Laboratorio de Suelos se trabajó la construcción del mapa de características edáficas de cada uno de los sectores diferenciados, exponiéndonos las capacidades nutricionales y los potenciales productivos.

La data climatológica, del distrito de Cacatachi, fue recopilada de la Dirección Regional del Servicio Nacional de Meteorología e Hidrología- SENAMHI - San Martín, la misma que corresponde a la base climatológica de la Estación Meteorológica de Tarapoto, por encontrarse en el radio de observación.

\section{Resultados y discusiones}

\subsection{Construcción del programa libre de SIG el para el análisis espacial del distrito de Cacatachi}

La herramienta SIG de carácter libre que se utilizó en el procesamiento de los datos geoespaciales fue el Programa denominado Quantun GIS, en su versión 2.4; con este programa se realizó la composición de los mapas para el distrito de Cacatachi. Para su uso no exige adquirir una licencia o permiso, se lo baja o instala libremente por la web sin otros protocolos.

La información que se acondicionó en función a los límites políticos del distrito de Cacatachi, fueron los temáticos de las características físicas de la ZEE Cumbaza, tales como la capa fisiográfica, suelos, capacidad de uso de los suelos y uso actual de las tierras que ejecutó la 
Dirección de Medio Ambiente del Proyecto Especial Huallaga Central Bajo Mayo, en el año 2009.

\subsection{Mapas generados y su descripción}

La información que se detalla, corresponde a algunos estudios temáticos elaborados para la meso ZEE de la Sub Cuenca del Río Cumbaza (Gobierno Regional de San Martín (2007.), que fueron acondicionado para el distrito de Cacatachi, que forma parte de esta unidad funcional de gestión.

Mapa 1. Fisiografía del distrito de Cacatachi: el mapa 1, indica la predominancia de Montañas y Valles Intramontanos de relieve plano en un 37\%, 1 688,62 ha, áreas planas depresionadas de origen coluvio aluvial, con drenaje de bueno a moderado, que permite desarrollar cultivos como el arroz, maíz etc. Mientras que las Montañas Altas de laderas moderadamente inclinadas se observan en un $21 \%$ con 943,78 ha, con relieve relativamente plano, pendientes que van de 4 a $8 \%$, suelos profundos a moderadamente profundos. Tiene Colinas altas ligera a moderadamente disectadas con un 14\%, 629,90 ha; colinas que presentan cimas sub-redondeadas y laderas cortas con pendientes entre 40 y $60 \%$. Montañas altas de laderas irregulares de modo empinado el 10,83\% con 494,46 ha; cimas de montañas colinadas representa el 8,20 \% con 374,51 ha; Montañas altas de laderas moderada inclinada el 4,01\% con 182,90 ha; Montañas bajas de laderas empinadas el 1,49 \% con 68,21 ha; Montañas valle intramontano de pendiente muy empinada el $1,12 \%$ con 50,96 ha, Montaña con valles intramontano de pendiente moderadamente inclinada con $0,85 \%$ con 36,82 ha y la zona urbana con $0,84 \%$ igual a 38,54 ha.

Tabla 1.

Características fisiográficas del distrito de Cacatachi

\begin{tabular}{|c|c|c|c|}
\hline \multirow{2}{*}{ Símbolo } & \multirow{2}{*}{ Descripción } & \multicolumn{2}{|c|}{ Superficie } \\
\hline & & ha & $\%$ \\
\hline 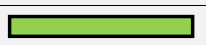 & Cimas con montañas colinadas & 374,51 & 8,20 \\
\hline 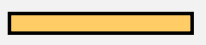 & Montañas Valles intramontado de relieve plano & 1688,62 & 36,98 \\
\hline & $\begin{array}{l}\text { Montañas Valles intramontado de pendiente } \\
\text { moderadamente inclinada. }\end{array}$ & 38,62 & 0,85 \\
\hline & $\begin{array}{l}\text { Montañas Valles intramontado de pendiente muy } \\
\text { empinada }\end{array}$ & 50,96 & 11,12 \\
\hline 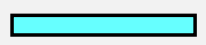 & Montañas altas de laderas moderadamente inclinadas & 182,90 & 4,01 \\
\hline$\square$ & Montañas altas de laderas moderadamente inclinadas & 943,78 & 20,67 \\
\hline & $\begin{array}{l}\text { Montañas altas de laderas irregulares moderadamente } \\
\text { inclinadas }\end{array}$ & 55,38 & 1,21 \\
\hline & $\begin{array}{l}\text { Montañas altas de laderas irregulares moderadamente } \\
\text { inclinadas }\end{array}$ & 494,46 & 10,83 \\
\hline コ & Montañas bajas de laderas empinadas & 68,21 & 1,49 \\
\hline$\square$ & Colinas altas ligeramente altas a disectadas & 629,90 & 13,80 \\
\hline 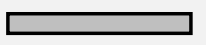 & Zona urbana & 38,58 & 0,84 \\
\hline Total área & & 4565,92 & 100,00 \\
\hline
\end{tabular}

Citar como: Castillo-Diaz, T., Lopez-Fulca, R., \& Ramirez-Navarro, W. (2021). Aplicación del GIS en la caracterización edafoclimática, del distrito de Cacatachi - provincia de San Martín, con fines de producción agrícola rentable y sostenible. Revista Agrotecnológica Amazónica, 1(2), 53-67. 


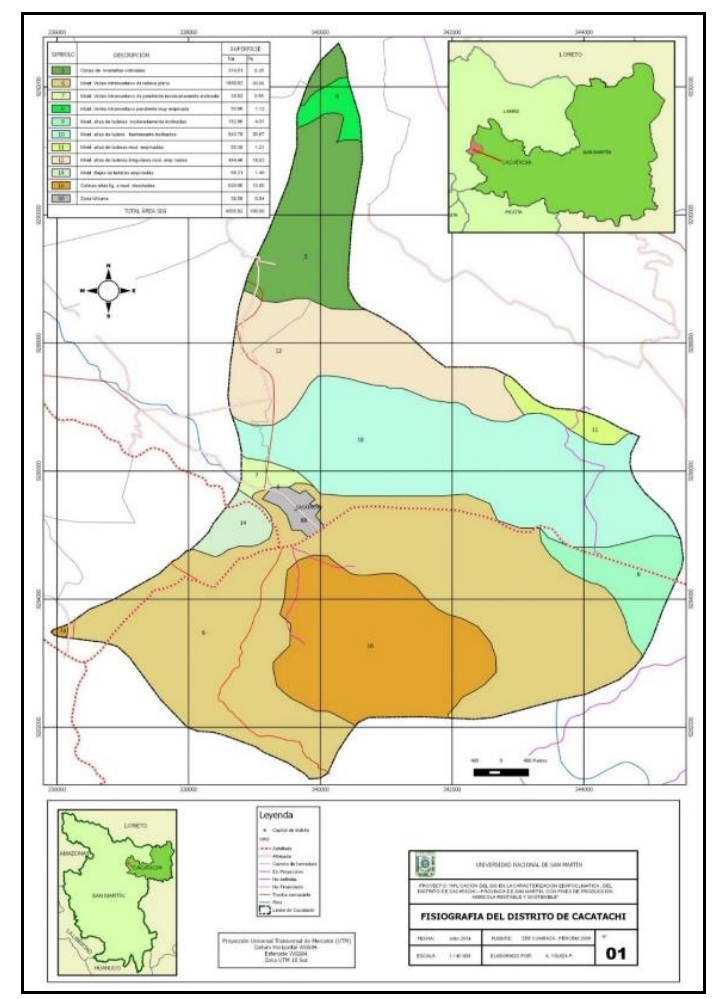

Figura 1: Fisiografía del distrito de Cacatachi

Mapa 2. Capacidad de uso de la tierra: el mapa 2 de Capacidad de uso de la Tierra, nos indica que en el distrito de Cacatachi predominan las Tierras aptas para cultivos en limpio con $36,98 \%$ y con 1 682,62 ha; tierras aptas para cultivos permanentes y pasto de Calidad Agroecológica media con limitaciones por pendiente, suelo y clima; asociados con tierras de producción forestal en un 24,68\%, con 1 126,68 ha; de Tierras aptas para cultivo permanente y pasto de Calidad Agroecológica media con limitaciones por pendiente, suelo y clima; asociados con tierras de protección por pendiente y suelos, en un 20,53\%, con 937,18 ha; como Tierras aptas para cultivo permanente de calidad Agroecológica baja con limitaciones por pendiente, suelo y clima; asociados con tierras aptas para pasto con limitaciones por pendiente, suelo y clima, en un $13,72 \%$, con 626,49 ha; tal como se muestra en la siguiente tabla. 
Tabla 2.

Características de capacidad de uso de las tierras el distrito de Cacatachi

\begin{tabular}{|c|c|c|c|}
\hline \multirow{2}{*}{ Símbolo } & \multirow{2}{*}{ Descripción } & \multicolumn{2}{|c|}{ Superficie } \\
\hline & & ha & $\%$ \\
\hline & $\begin{array}{l}\text { Tierras aptas para cultivo en limpio de Calidad Agrológica media con } \\
\text { limitaciones en el suelo y clima. }\end{array}$ & 1688,62 & 36,98 \\
\hline & $\begin{array}{l}\text { Tierras aptas para cultivo permanente de Calidad Agrológica media } \\
\text { con limitaciones por pendiente, suelo y clima - Asociados con tierras } \\
\text { aptas para pastos de calidad agrológica media con limitaciones de } \\
\text { pendiente, suelo y clima. }\end{array}$ & 3,41 & 0,07 \\
\hline & $\begin{array}{l}\text { Tierras aptas para cultivo permanente de Calidad Agrológica media } \\
\text { con limitaciones por pendiente, suelo y clima - Asociados con tierras } \\
\text { de protección por pendiente y suelo. }\end{array}$ & 94,00 & 2,06 \\
\hline & $\begin{array}{l}\text { Tierras aptas para cultivo permanente de Calidad Agrológica baja con } \\
\text { limitaciones por pendiente, suelo y clima - Asociados con tierras } \\
\text { aptas para pastos con limitaciones por pendiente, suelo y clima. }\end{array}$ & 626,49 & 13,72 \\
\hline & $\begin{array}{l}\text { Tierras para protección por pendiente y suelo - Asociados a Tierras } \\
\text { aptas para cultivo permanente de Calidad Agrológica media con } \\
\text { limitaciones por pendiente, suelo y clima. }\end{array}$ & 50,96 & 1,12 \\
\hline & $\begin{array}{l}\text { Tierras aptas para cultivo permanente y pasto de Calidad Agrológica } \\
\text { media con limitaciones por pendiente, suelo y climas - Asociados } \\
\text { con tierras de protección por pendiente y suelos. }\end{array}$ & 937,18 & 20,53 \\
\hline & $\begin{array}{l}\text { Tierras aptas para cultivo permanente y pasto de Calidad Agrológica } \\
\text { media con limitaciones por pendiente, suelo y climas - Asociados } \\
\text { con tierras de producción forestal. }\end{array}$ & 1126,68 & 24,68 \\
\hline - & Zona urbana & 38,58 & 0,84 \\
\hline Total área & SIG & 4565,92 & 100,00 \\
\hline
\end{tabular}

Fuente: Gobierno Regional de San Martín (2007), meso ZEE Cumbaza

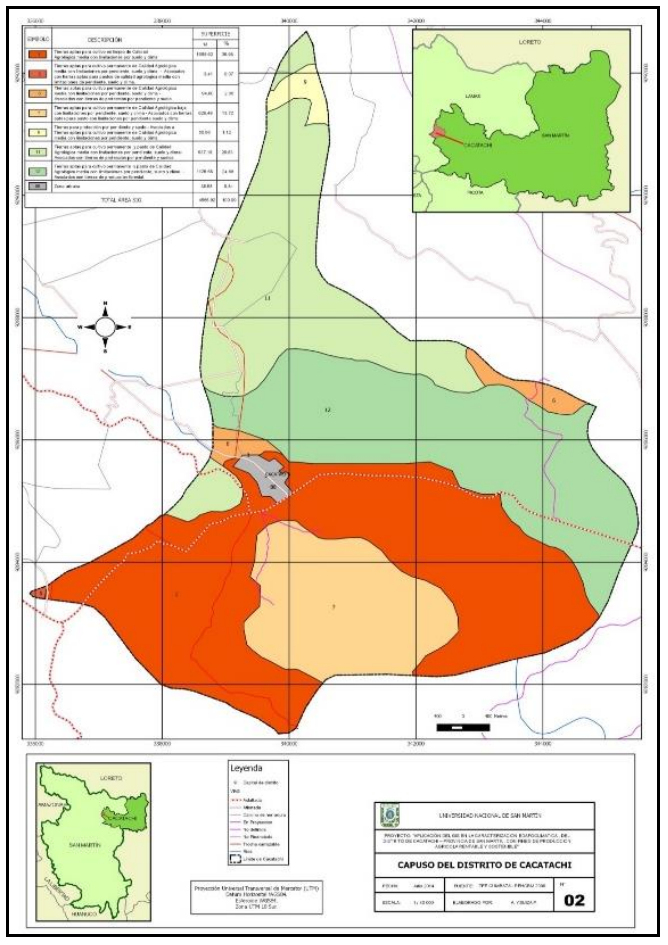

Figura 2. Características de capacidad de uso de las tierras el distrito de Cacatachi

Citar como: Castillo-Diaz, T., Lopez-Fulca, R., \& Ramirez-Navarro, W. (2021). Aplicación del GIS en la caracterización edafoclimática, del distrito de Cacatachi - provincia de San Martín, con fines de producción agrícola rentable y sostenible. Revista Agrotecnológica Amazónica, 1(2), 53-67. 
Mapa 3. Características de los suelos del distrito de Cacatachi: el mapa 3, de las características de suelos del distrito de Cacatachi, muestra una superficie de 4565,92 ha, que se encuentra distribuido de la siguiente manera: un $37 \%$ pre dominan los suelos Typic Ustorthents que representa el 1 688,62 has ,seguido de los Suelos Typic Eutrustepsts-Lithic Dystrustepts en un $21,52 \%$ equivalentes a 982,39 ha, en el orden le sigue suelos Vertic Dystrusteps en un 14\% con 626,49 ha, mientras que Typic Dystrustepts -Lithic Dystrustepts con 429,89 ha, Typic Dystrustepts con 182,90 ha, Lithic Calciustepts-Miscelaneo con 50,96 ha la zona urbana con 38,58 ha y entre otros tipos de suelos tal como se muestra en la siguiente tabla.

Tabla 3.

Características del suelo del distrito de Cacatachi

\begin{tabular}{|c|c|c|c|}
\hline \multirow{2}{*}{ Símbolo } & \multirow{2}{*}{ Soil Taxon } & \multicolumn{2}{|c|}{ Superficie } \\
\hline & & ha & $\%$ \\
\hline$\square$ & Typic Ustorthents & 1688,62 & 36,98 \\
\hline$\square$ & Typic Calciustepts & 3,41 & 0,07 \\
\hline$\sqsupset$ & Typic Dystrusteps & 182,90 & 4,01 \\
\hline$\sqsupset$ & Vertic Dystrusteps & 626,49 & 13,72 \\
\hline$\sqsupset$ & Typic Eutrustepts - Lithic Dystrusteps & 982,39 & 21,52 \\
\hline$\square$ & Typic Dystrusteps - Lithic Dystrusteps & 429,89 & 9,42 \\
\hline 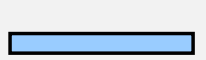 & Lithic Calciustepts - Miscekan & 50,96 & 1,12 \\
\hline$\square$ & Lithic Dystrusteps - Lithic Calciustepts & 562,67 & 12,32 \\
\hline & Zona urbana & 38,58 & 0,84 \\
\hline \multicolumn{2}{|c|}{ Total área SIG } & 4565,92 & 100,00 \\
\hline
\end{tabular}

Fuente: Gobierno Regional de San Martín (2007), meso ZEE Cumbaza

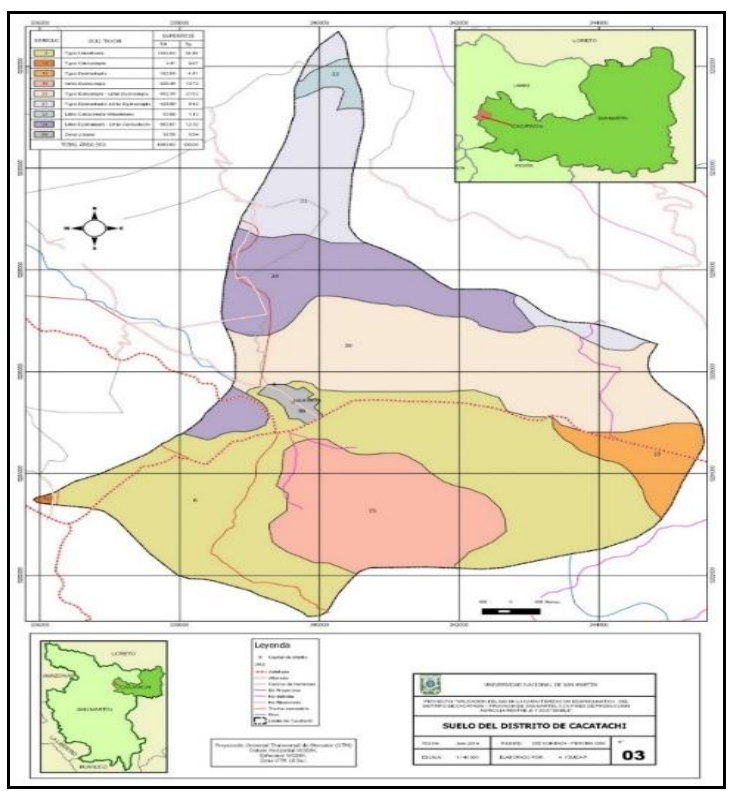

Figura 3. Características de los suelos del distrito de Cacatachi

Citar como: Castillo-Diaz, T., Lopez-Fulca, R., \& Ramirez-Navarro, W. (2021). Aplicación del GIS en la caracterización edafoclimática, del distrito de Cacatachi - provincia de San Martín, con fines de producción agrícola rentable y sostenible. Revista Agrotecnológica Amazónica, 1(2), 53-67. 
Mapa 4. Uso actual de las tierras del distrito de Cacatachi: el mapa 4, de Uso actuales, indica que más del 50\%, del territorio del distrito de Cacatachi está conformado por Bosque antrópico, entre purmas y cultivos intensivos que equivale a 2327,46 ha; el $30 \%$ corresponde a Bosque Antrópico, con cultivos Intensivos bajo Riego, representando 1371,73 ha; mientras que el 9,49 \% corresponde a Bosque Antrópico de Zonas degradadas con 433,24 ha, mientras que el área ocupada por Bosque Antrópico - producción de uva y cacao está ocupada en un 3,58 \%, con 163,65 ha, con bosques secundarios abarca el 3,59\%, con 164,01 ha; con 0,50 \%, 22,80 ha; entre otras características que se observa en la siguiente tabla, ver mapa.

Tabla 4.

Características de uso actual - distrito de Cacatachi

\begin{tabular}{|c|c|c|c|}
\hline \multirow{2}{*}{ Símbolo } & \multirow{2}{*}{ Descripción } & \multicolumn{2}{|c|}{ Superficie } \\
\hline & & ha & $\%$ \\
\hline$\square$ & Bosque Secundario & 164,01 & 3,59 \\
\hline & Bosque Antrópico - Cultivos Estacional en BST & 22,80 & 0,50 \\
\hline$\square$ & Bosque Antrópico - Cultivos Intensivos de Bajo Riesgo & 371,73 & 30,04 \\
\hline$\square$ & Bosque Antrópico - Pumas y Cultivos Intensivos & 2327,46 & 50,97 \\
\hline$\square$ & Bosque Antrópico - Zonas Degradadas & 433,24 & 9,49 \\
\hline$\square$ & Pastos & 9,45 & 0,21 \\
\hline$\square$ & Sistema Agroforestal & 0,77 & 1,12 \\
\hline 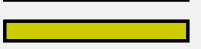 & Bosque Antrópico - Producción de uva y cacao & 163,65 & 3,58 \\
\hline$\square$ & Zona urbana & 38,58 & 0,84 \\
\hline Total área & SIG & 4565,92 & 100,00 \\
\hline
\end{tabular}

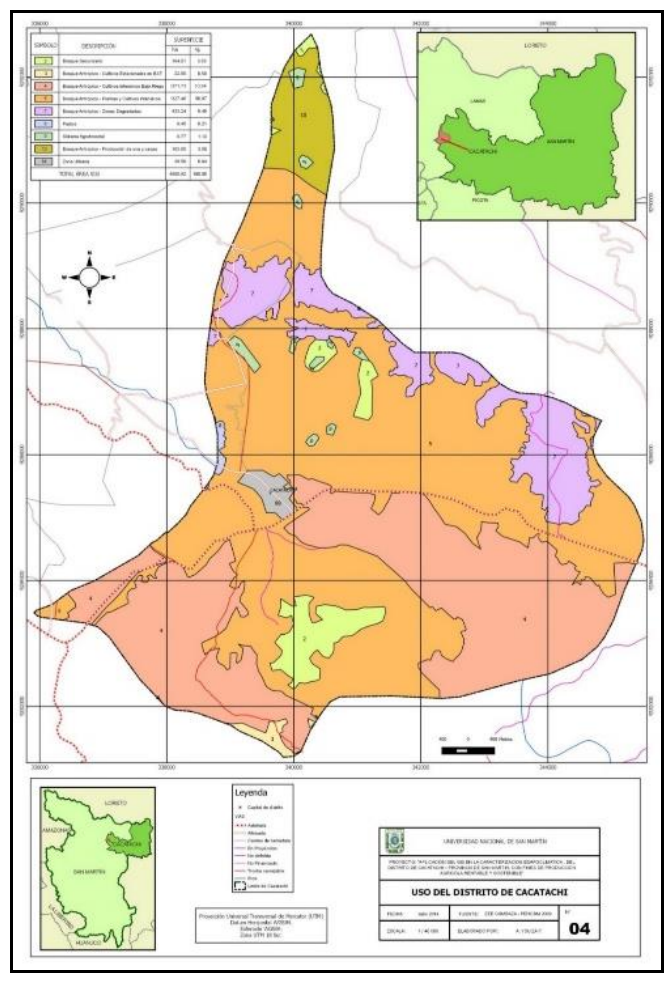

Figura 4. Usos actuales de las tierras del distrito de Cacatachi

Citar como: Castillo-Diaz, T., Lopez-Fulca, R., \& Ramirez-Navarro, W. (2021). Aplicación del GIS en la caracterización edafoclimática, del distrito de Cacatachi - provincia de San Martín, con fines de producción agrícola rentable y sostenible. Revista Agrotecnológica Amazónica, 1(2), 53-67. 


\section{Conclusiones}

Se creó una base de datos en Programa Quantum GIS (QGIS) que permite el manejo de la información edafoclimática, en base a la información geomorfológica, capacidad de uso de los suelos, a las características físicas químicas de los suelos y además se contrapusieron el mapa de los actuales usos de las tierras, en función a lo cual, producto del análisis y modelamiento de las capas temáticas, más el descriptivo para ubicar las diferentes calicatas, el sistema nos genera un total de 104 mapas, en detalle, cuyas representaciones espaciales nos exponen la caracterización edáfica y climática, considerando las zonas estratificadas en el proyecto, donde se hicieron las calicatas, de los cuales se hicieron luego en al Laboratorio de suelos, los respectivos análisis de las características químicas, físicas y biológicas de los horizontes identificados en los perfiles.

Se generaron los siguientes mapas temáticos: mapa fisiográfico, que describe las caracte-rísticas del relieve fisiográfico del distrito de Cacatachi; mapa de la capacidad de uso de las tierras, que explica las potencialidades que presentan las tierras en función a su capacidad de uso mayor; mapa de las características del suelo, que detalla las condiciones de los suelos y mapa del uso actual de las tierras del distrito de Cacatachi.

El resultado de esta caracterización edafoclimática brinda diferentes alternativas a los decisores considerando otros aspectos como: inversión en siembras, aplicación de tecnologías, sistemas de riego, uso de variedades mejoradas, así como también la articulación a mercados estables.

La composición cartográfica estructurada, nos presenta datos procesados en el laboratorio que representan características físico químicas del área de estudio, entre ambas suman gran cantidad de información, el reto es, como entre si se conectan o relacionan, como de un determinado espacio visualizo indicadores espaciales y por otro lado diferentes datos como son el $\mathrm{PH}$, clima y coordenadas.

Los mapas generados se convierten en herramientas claves para la planificación en las sucesivas campañas de siembras de los principales cultivos, es decir como una plataforma integradora y orientadora de lo que se debe sembrar y sobre todo en que zonas del distrito se debe practicar agricultura de riego, con cultivos permanentes, con pasturas y además que zonas o espacios no tienen más capacidades que las de protección.

\section{Agradecimientos}

A la Universidad Nacional de San Martín - Tarapoto, por el financiamiento del proyecto de investigación a partir de cuyos resultados se genera el presente artículo. 


\section{Referencias bibliográficas}

Aguirre-Forero, S. E., Piraneque-Gambasica, N. V., \& Vásquez-Polo, J. R. (2018). Características edáficas y su relación con usos del suelo en Santa Marta, Colombia. Entramado, 14(1), 242-250. https://www.redalyc.org/journal/2654/265457559017/html/

Giannini Kurina, F., Hang, S., Cordoba, M. A., Negro, G. J., \& Balzarini, M. G. (2018). Enhancing edaphoclimatic zoning by adding multivariate spatial statistics to regional data. Geoderma, 310, 170-177. https://doi.org/10.1016/j.geoderma.2017.09.011

GORESAM. (2009). Las potencialidades y limitaciones del departamento de San Martín. http://siar.regionsanmartin.gob.pe/documentos/potencialidades-limitaciones-departamentosan-martin-zonificacion

GORESAM. (2020). San Martín es la sexta región con mayor crecimiento de sus exportaciones. https://www.regionsanmartin.gob.pe/Noticias?url=noticia\&id=6143

Kahveci, M. (2017). Contribution of GNSS in precision agriculture. Proceedings of 8th International Conference on Recent Advances in Space Technologies, RAST 2017, $513-$ 516. https://doi.org/10.1109/RAST.2017.8002939

Laurett, R., Paço, A., \& Mainardes, E. W. (2021). Sustainable development in agriculture and its antecedents, barriers and consequences - an exploratory study. Sustainable Production and Consumption, 27, 298-311. https://doi.org/10.1016/j.spc.2020.10.032

Lesmo Duarte, N. D., Ferreira da Silva, A., Schlindwein, M. M., \& Solis Mendoza, B. A. (2018). Agricultura Familiar Campesina: un análisis de la producción de cultivos tradicionales en el Departamento de Concepción - Paraguay. Investigación Agraria, 20(2), 136-142. https://doi.org/10.18004/investig.agrar.2018.diciembre.136-142

Longley, P. A., \& Frank Goodchild, M. (2020). Geographic Information Science and Systems. In International Encyclopedia of Human Geography (Segunda Ed, pp. 29-36). https://doi.org/10.1016/b978-0-08-102295-5.10557-8

Lyford, J. (2017). Food security in the developing world. Journal of Nutrition Education and Behavior, 49(5), 451. https://doi.org/10.1016/j.jneb.2017.01.010

Mani, P. K., Mandal, A., Biswas, S., Sarkar, B., Mitran, T., \& Meena, R. S. (2021). Remote Sensing and Geographic Information System: A Tool for Precision Farming. In Geospatial Technologies for Crops and Soils (pp. 49-111). https://doi.org/10.1007/978-981-15-68640_2

Mok, W. K., Tan, Y. X., \& Chen, W. N. (2020). Technology innovations for food security in Singapore: A case study of future food systems for an increasingly natural resource-scarce world. Trends in Food Science and Technology, 102, 155-168.

https://doi.org/10.1016/j.tifs.2020.06.013

Moysiadis, V., Sarigiannidis, P., Vitsas, V., \& Khelifi, A. (2021). Smart Farming in Europe. 
Computer Science Review, 39, 100345. https://doi.org/10.1016/j.cosrev.2020.100345

Nabati, J., Nezami, A., Neamatollahi, E., \& Akbari, M. (2020). GIS-based agro-ecological zoning for crop suitability using fuzzy inference system in semi-arid regions. Ecological Indicators, 117, 106646. https://doi.org/10.1016/j.ecolind.2020.106646

Palomino, W., Morales, G., Huaman, S., \& Telles, J. (2018). PETEFA: Geographic Information System for Precision Agriculture. Proceedings of the 2018 IEEE 25th International Conference on Electronics, Electrical Engineering and Computing, INTERCON 2018, 14. https://doi.org/10.1109/INTERCON.2018.8526414

Perez-Garcia, C. A., Pérez-Atray, J. J., Hernández -Santana, L., Gustabello-Cogle, R., \& Becerra-de Armas, E. (2019). Sistema de Información Geográfica para la agricultura cañera en la provincia de Villa Clara. Revista Cubana de Ciencias Informáticas, 13(2), 30-46. https://rcci.uci.cu/?journal=rcci\&page $=$ article $\&$ op $=$ view $\&$ path $\% 5 B \% 5 D=1796$

Rizaldi, T., Putranto, H. A., Riskiawan, H. Y., Setyohadi, D. P. S., \& Riaviandy, J. (2019). Decision support system for land selection to increase crops productivity in Jember Regency Use Learning Vector Quantization (LVQ). Proceedings - 2019 International Conference on Computer Science, Information Technology, and Electrical Engineering, ICOMITEE 2019, 82-85. https://doi.org/10.1109/ICOMITEE.2019.8921033

Saing, Z., Djainal, H., \& Deni, S. (2021). Land use balance determination using satellite imagery and geographic information system: case study in South Sulawesi province, Indonesia. Geodesy and Geodynamics, 12(2), 133-147. https://doi.org/10.1016/j.geog.2020.11.006

Stojanovic, M. (2019). Biomimicry in agriculture: is the Ecological System-Design Model the Future Agricultural Paradigm? Journal of Agricultural and Environmental Ethics, 32, 789-804. https://doi.org/10.1007/s10806-017-9702-7

Sun, Z., Wang, D., \& Zhong, G. (2018). Extraction of farmland geographic information using OpenStreetMap data. 2018 7th International Conference on Agro-Geoinformatics, AgroGeoinformatics 2018. https://doi.org/10.1109/Agro-Geoinformatics.2018.8476088 Zhang, F., \& Cao, N. (2019). Application and research progress of Geographic Information System (GIS) in agriculture. 2019 8th International Conference on Agro-Geoinformatics, Agro-Geoinformatics 2019. https://doi.org/10.1109/Agro-Geoinformatics.2019.8820476

\section{Conflicto de intereses}

Los autores declaramos que no existen conflictos de interés. 


\section{Contribuciones de los autores}

Castillo-Diaz, Tedy: Coordinación del proyecto y análisis estadísticos e interpretación.

Lopez-Fulca, Roaldo: Redacción y parte metodológica.

Ramirez-Navarro, Wiliams: Revisión final del artículo. 